\title{
Myths and Misconceptions about Intrauterine Devices: Impact on Usage
}

\author{
Emmanuel Lamptey ${ }^{\mathrm{a}^{*}}$, Michael A. Okunlola ${ }^{\mathrm{b}}$, Adesina Oladokun ${ }^{\mathrm{b}}$ \\ ${ }^{a}$ Institute of Life and Earth Sciences (Including Health and Agriculture), Pan African University, University of Ibadan, Nigeria. \\ ${ }^{b}$ Department of Obstetrics and Gynecology, University College Hospital, University of Ibadan, Ibadan, Nigeria.
}

Received 12 June 2020; Accepted 18 October 2020

\begin{abstract}
The Intrauterine Devices (IUDs) have the benefit of providing long-term protection against unwanted pregnancies. This study investigates the myths and misconceptions associated with IUDs and their influence on the usage. This crosssectional was conducted in six family planning centres in Accra. The questionnaire included statements of misconceptions about IUDs and participants responded either in agreement or in disagreement with each of the statements. Three hundred eligible respondents completed the questionnaire. The study commenced on January 2019 and ended on June 2019. We observed a heightening level of misconceptions about IUDs among the participants. Pain during insertion $(68.7 \%)$, the male partner feeling it during intercourse (66.7\%), IUDs breeding infections (66.3\%) and the belief that it can damage the womb $(65.7 \%)$ were the major misconceptions identified about the device. Although, these misconceptions may be the true reflection of the experiences of these users, an accurate knowledge about the device is still lacking which can influence the potential patronage. Massive education to change and dispels these misconceptions are needed.
\end{abstract}

Keywords: Myths and Misconceptions; Intrauterine Devices; Impact; Usage.

\section{Introduction}

Many sexually active women of reproductive age rely on long-acting contraceptives to prevent unwanted pregnancies primarily because of its effectiveness, long-term protection and convenient use. Long-acting contraceptives do not depend on the sexual behaviors of its users and unwanted pregnancies are well-controlled [1]. Unwanted pregnancies and abortions remain a serious reproductive health challenge confronting women as unwanted pregnancies remain at 40\% globally [2]. The Ghana Demographic and Health Survey 2014 report showed that $39.0 \%$ of women around the age of 20 years have at least a child.

The Intra-Uterine Devices (IUDs), an easily reversible, safe and highly effective long-acting method on the market today is serving the key purpose of preventing unintended and high-risk pregnancies among women. It is estimated that $12 \%$ of all reproductive-aged women are using the IUD worldwide [3]. In Europe, 50\% of contraceptive users are on IUD and in China; the figure approaches 33\% [3]. IUDs have been overwhelmingly documented and recommended as safe for use in women by the World Health Organization, the America College of Obstetrics and Gynecology and, the American Association of Pediatrics. Information released by the America College of Obstetrics and Gynecology stated that the device should be offered to nulliparous and parous adolescents [4].

In Ghana, the device (IUD) is readily available through the Ghana Health Service (GHS), Ghana Social Marketing

\footnotetext{
* Corresponding author: emmanuelamptey88@gmail.com
}

\section{doi) http://dx.doi.org/10.28991/SciMedJ-2020-0204-3}

$>$ This is an open access article under the CC-BY license (https://creativecommons.org/licenses/by/4.0/).

(C) Authors retain all copyrights. 
Foundation (GSMF), Ghana Registered Midwives Association (GRMA), and Planned Parenthood Association of Ghana (PPAG) clinics. However, Patronage from these sources indicated a $40 \%$ decline in the provision of the IUD from 25,000 acceptors in 1995 to only 10,000 in 2000(Ghana Demographic Health Survey, 2000). There are several explanations offered why there is a decline in the patronage of the device.

The plausible explanation for the observed decline in usage includes misconceptions, low publicity, infections, risk of ectopic pregnancies and infertility [5]. Misconceptions cause low demand and uptake of contraceptives in general and are one of the keys reasons for the poor performance of the device because they spread rapidly by a conversation among women and through social networks [5].

Thapa et al. (2019) in their analysis described these misconceptions as subjective norms that affect the intentions and behaviors of women to use the device in Nepal [6]. Women are discouraged from using the device due to the family and society misconceptions even though; Family and society are not the only sources of these myths

Most recently, a study was done in China, Wang et al. 2020 discovered some of these beliefs are common among healthcare providers. Most health care providers do not have adequate knowledge regarding the safety and effectiveness of the device. Conservative beliefs among the healthcare providers stemmed from complication associated with using the device [1]. Healthcare providers also perceive that the device has complications such as Pelvic Inflammatory Diseases (PIDs), Infertility and painful insertions according to a study in Brazil [7].

Therefore, Healthcare providers sometimes become barriers, fueling these misapprehensions [8]. Besides, there are still unfounded beliefs among young women regarding the device. Payne et al. (2016) identified fear of infertility later in life and harm as some of them [9]. In addition, Elkhateeb et al. (2020) investigated the concept in nulliparous Egyptian women and found that fear of infections and subsequent infertility were the main negative impressions [10].

Religious restrictions have partly contributed to these misconceptions. In the situation where long-term contraceptives are affordable for sexually active women, many do not utilize them because of moral teachings and guidance by some religious organizations in society. Example of which can be found in the US, where the healthcare market is controlled by Catholics [11]. Women coming from such religious background hardly patronize IUDs.

Cultural barriers, poor access, forgetfulness and low educational attainment, negative stereotypes against women patronizing the device in Sub-Sahara Africa are some of the drivers of this low utilization [12]. In a country like Nigeria with the largest population in Africa, these misconceptions associated with the device can be found in $33-57 \%$ of the population [12]. Some couples will never like to use the device because they believe IUDs do cause pelvic inflammatory diseases, pain, ectopic pregnancies and a host of others.

A Study by Goller et al. (2020) indicated that out of 1000 women using IUDs yearly only 1.6 cases contract PIDs, meaning PIDs associated with the device has significantly reduced if not mitigated. In addition, a recent finding revealed that the levonorgestrel IUD, when prescribed for medical purposes, relieves menstrual bleeding difficulties and pain in $80 \%$ of its users [13].

Some other statements like the male partners can feel the device; it breeds infections and damages the womb etc. are attributable factors to the low patronage of IUDs. The Historic Dalkon Shield event and other related side effects of the device have fueled these misconceptions. Since contraceptive choices in women are influenced by personal circumstances and perceptions, the current study investigated these misconceptions from the viewpoints of IUD users and examined whether they influence the uptake of the device.

\section{Materials and Methods}

This study employed a cross-sectional survey method to recruit 300 women at six randomly selected family planning centres in Accra from January-June 2019. Women aged 20-49 and are currently using the device were considered eligible. The study excluded women with a history of depression and sexual inactivity to prevent their experiences from influencing their responses and the outcome of this study.

The Ghana Health Service Ethics Committee reviewed and approved the study and all ethical processes dully followed. Respondents were fully informed about the purpose of the study and participation was voluntary. Their informed consents were obtained before administering the questionnaire. The questionnaires consist of rumours, beliefs and negative statements reviewed from current and existing works of literature concerning IUDs. Respondents were required to show their level of agreements with these myths and misconceptions about IUDs. Responses to the various statements were categorized into Agree, Disagree and Neutral (No idea). The raw data were entered and cleaned using Microsoft Excel. Statistical analysis was performed with SPSS version 20. A chi-square test (for misconceptions and the use of IUD) was computed with significance level stated as a p-value. The confidence level was $95 \%$ and Statistical significance set to be $\mathrm{p}<0.05$. 


\section{Results}

The majority of the participants (34\%) were aged 26-30 years, followed by those within 31-35 years (31.7\%) and the least represented age group was $46-49$ years (3\%). Almost $60 \%$ of the participants were married (179/300) and $38.3 \%$ had finished secondary school. Approximately $81 \%$ of the women were parous while the remaining $19.4 \%$ had never given birth. Christians formed $57.6 \%$ of the total participants. Eighty-eight per cent (264/300) of the respondents stated that they would not use the device again.

Table 1. Demographic characteristics of Respondents

\begin{tabular}{cc}
\hline Characteristics & $\mathbf{n}(\%)$ \\
\hline Age group & $29(9.7 \%)$ \\
$20-25$ & $102(34.0 \%)$ \\
$26-30$ & $95(31.7 \%)$ \\
$31-35$ & $48(16.0 \%)$ \\
$36-40$ & $25(8.3 \%)$ \\
$41-45$ & $1(3 \%)$ \\
$46-49$ & \\
Marital Status & $179(59.7 \%)$ \\
Married & $6(2.0 \%)$ \\
Single & $115(38.3 \%)$ \\
In union but not married & \\
Educational level & $28(3.3 \%)$ \\
No formal education & $45(15.0 \%)$ \\
Primary & $115(38.3 \%)$ \\
Secondary & $112(37.3 \%)$ \\
Tertiary & \\
Religion & $80(26.7 \%)$ \\
Islam & $47(15.7 \%)$ \\
Traditional/Others & $173(57.6 \%)$ \\
Christian & \\
Parity & $58(19.4 \%)$ \\
Nulliparous & $242(80.6 \%)$ \\
Parous & \\
Yill Ever Use the Device & \\
No & \\
\hline & \\
\hline & \\
\hline & \\
\hline & \\
\hline
\end{tabular}

Twelve (12) statements on various misconceptions about IUDs were gathered from rumors, beliefs, reviewed works of literature and presented to the respondents. The responses to each of the statement are shown in Table 2 . The percentage responses to the agreement with each statement on myths and misconception range from 24.7-68.7\%. The majority of the participant agreed to the statements: the IUD is painful to have it inserted(68.7\%), the male partner can feel the device during sexual intercourse(66.7\%),IUDs breed infections(66.3\%) and there is a good chance that the device can damage the womb(65.7\%). Furthermore, $64.7 \%$ of the respondents believed that the device can rust inside them and, $63.3 \%$ agreed the IUD is capable of moving inside the body.

However, the participants disagreed with some of these myths and misconceptions. Almost 40\% do not support the statement that IUDs are not suitable if a woman has had more than three children. According to these women, IUDs are useful irrespective of parity. Then $26.3 \%$ of the women also disagreed that conception is difficult after using IUD for a time. Lastly, $26.0 \%$ of respondents do not believe the statement that IUDs are only usable in older women. The percentages of women who neither agreed nor disagreed or had neutral views with each statement ranged 15.0-36\%. 
Table 2. Responses to the Statements

\begin{tabular}{llll}
\hline \multicolumn{1}{c}{ Statements } & Responses (n (\%)) & \\
\hline & Agree & $\begin{array}{c}\text { Neutral } \\
\text { (I don't know) }\end{array}$ & Disagree \\
\hline 1. The male partner can feel the IUD during sexual intercourse & $200(66.7)$ & $45(15.0)$ & $55(18.3)$ \\
2. IUDs are associated with pain during sexual intercourse & $182(60.7)$ & $47(15.7)$ & $71(23.7)$ \\
3. If one falls pregnant on IUD, the baby will come out holding it. & $169(56.3)$ & $74(24.7)$ & $57(19.0)$ \\
4. Conception is difficult after using an IUD for a time. & $162(54.0)$ & $59(19.7)$ & $79(26.3)$ \\
5. Using IUD is the same as committing abortion so it is not right for certain religion & $180(60.0)$ & $55(18.3)$ & $65(21.7)$ \\
6. It is the breeding place for infections. & $199(66.3)$ & $57(19.0)$ & $44(14.7)$ \\
7. The IUD is painful to have it inserted & $206(68.7)$ & $46(15.3)$ & $48(16.0)$ \\
8. It can rust inside you & $194(64.7)$ & $57(19.0)$ & $49(16.3)$ \\
9. The IUD can only be used in older women & $163(54.3)$ & $59(19.7)$ & $78(26.0)$ \\
10. There is good chance that the IUD can damage the womb & $197(65.7)$ & $56(18.7)$ & $47(15.7)$ \\
11. It can move inside your body & $190(63.3)$ & $58(19.3)$ & $52(17.3)$ \\
12. It is not suitable if you have had more than 3 children. & $74(24.7)$ & $108(36)$ & $118(39.3)$
\end{tabular}

Table 3 shows that there is a statistically significant relationship between Five (5) of these Statements and the future use of the device. Statements such as pain during insertion, male partner feeling the device during sex, a breeding place for infections, damaging the womb and pains felt during sexual intercourse made significant differences and influenced the uptake of the device. Participants who agreed that IUDs are painful to be inserted are less likely to use the device in the future $\left(\mathrm{X}^{2}=10.147, \mathrm{p}=0.006\right)$. Also, participants who agreed to the statement that the male partner can feel the device during sex were less likely to use the device in the future $\left(X^{2}=6.984, p=0.030\right)$. The statement IUDs serves as a breeding place for infection was statistically associated with the respondents' decision to use the device in the future $\left(X^{2}=9.345, p=0.009\right)$. Respondents who agreed that IUDs can damage the womb had a high probability of not using the device ever again $\left(\mathrm{X}^{2}=10.038, \mathrm{p}=0.007\right)$ and lastly respondents who think IUDs usage is associated with pain during sexual intercourse were not likely to use the device in the future $\left(\mathrm{X}^{2}=13.773\right.$, $\mathrm{p}=$ $0.001)$.

Table 3. Potential Association between the Five (5) Statements regarding IUDs and the use of the device

\begin{tabular}{|c|c|c|c|c|c|}
\hline \multirow{2}{*}{ Variable } & \multicolumn{2}{|c|}{ Will Ever Use IUD } & \multirow{2}{*}{$\mathbf{X}^{2}$} & \multirow{2}{*}{ df } & \multirow{2}{*}{$P$ value } \\
\hline & No & Yes & & & \\
\hline \multicolumn{6}{|l|}{ Painful to have it inserted } \\
\hline Agree & 173 & 33 & 10.147 & 2 & 0.006 \\
\hline Disagree & 46 & 2 & & & \\
\hline Neutral & 45 & 1 & & & \\
\hline \multicolumn{6}{|c|}{ Male Partner can feel the device during sex } \\
\hline Agree & 169 & 31 & 6.984 & 2 & 0.030 \\
\hline Disagree & 52 & 3 & & & \\
\hline Neutral & 43 & 2 & & & \\
\hline \multicolumn{6}{|l|}{ Breeding place for Infections } \\
\hline Agree & 167 & 32 & 9.345 & 2 & 0.009 \\
\hline Disagree & 42 & 2 & & & \\
\hline Neutral & 55 & 2 & & & \\
\hline \multicolumn{6}{|l|}{ IUD can damage the Womb } \\
\hline Agree & 165 & 32 & 10.038 & 2 & 0.007 \\
\hline Disagree & 46 & 1 & & & \\
\hline Neutral & 53 & 3 & & & \\
\hline \multicolumn{6}{|l|}{ Pain during sexual intercourse } \\
\hline Agree & 150 & 32 & 13.773 & 2 & 0.001 \\
\hline Disagree & 68 & 3 & & & \\
\hline Neutral & 48 & 1 & & & \\
\hline
\end{tabular}

\section{Discussion}

The results showed that a large number of the respondents (24.7-68.7\%) agreed with some of the statements described as myths and misconception about Intrauterine conceptive. Only a few of them (14.7-39.3\%) disagreed with certain misconceptions. Respondents were able to disagree and debunked statements such as the IUD is not suitable if you have more than three children, it is not useful in older women and conception is difficult after using IUD for a 
time. The number of participants who disagreed was a little bit less than the number of women who neither agreed nor disagreed to these statements. Such category of participants remained neutral possibly because they do not have accurate and adequate knowledge about IUD contraceptives. This shows that IUD users still need more education about the device to dispel some of these uncertainties and misconceptions.

The topmost statement this present study identified was "IUDs are painful to have it inserted". This finding is similar to the study by Michie et al. (2014), whose studies investigated myths and misconceptions about intrauterine contraception among women seeking termination of pregnancy [14]. Surprisingly, these very statements have also been the concerns and beliefs of some reproductive healthcare providers. Elkhateeb et al. (2020) found in their studies these same beliefs among health professionals responsible for inserting the device in nulliparous women in Egyptian. In Brazil, a survey conducted among Gynaecologists revealed that the IUDs were perceived as difficult and painful to have it inserted [7].

However, a study by Manzouri et al. (2010) identified IUDs causing abortions and cancers as the most common misconception among women in Isfahan, Iran [15]. Therefore, there is an urgent need for family planning providers to address pain issues associated with the IUD insertion because it could be the real experiences of users. It is prudent that women should be prepared psychologically and be made aware of some of the side effects/ discomforts that come with the method. Family planning providers should constantly apply the appropriate insertion techniques to minimize the pain.

Most women still believe that the male counterpart can feel the IUD string during sexual intercourse. Again Manzouri et al. (2010) confirmed this as the second most popular misconception in their studies [15]. Proper insertion procedures will prevent this from happening and women should be well reassured.

IUD users also have the notion that the device breeds infections. This is not a new situation as research done in the United States stated that most women believe IUDs like other foreign objects lower the body's natural defences and open up for pelvic inflammatory diseases [16]. Nevertheless, there are many best practices put in place to minimize the risk of infection with Intrauterine Devices such as screening women with a history of infections and physical examination to identify those at risk of future infections. Users should also be educated that infections are likely to occur during the first 20 days and proper medications will manage that if necessary.

The study also investigated the influence of these misconceptions on IUD usage. Our findings discovered that certain misconceptions do influence the patronage of Intrauterine conceptive. There was a statistically significant relationship between five of these variables and the future use of IUD. Statements like the IUD is painful to have it inserted, male partners can feel the device during sex, it is a breeding place for infections, it can damage the womb and rust inside the body could influence future usage. These are issues of concern to women using the device, misinforming choices, which have an impact on the behaviours of users. In some women, these beliefs make more sense to them than any other information because it fits into their established opinions. These identified statements cause lack of understanding about IUDs leading to low utilization. It also creates the situation where women cannot see the value, use and purpose of the device in terms of safety, efficiency and convenience.

These misconceptions also push reproductive-aged women to make and take decisions that are not in their best interest and reproductive goals. For instant women with doubts about IUDs and their safety concerns end up using other less effective methods, which dispose them to an unplanned pregnancy and unsafe abortions.

Furthermore, women keeping the fear of permanent infertility and complications later in life are deterred from using the device and other potentials users upon hearing some of these misconceptions are discouraged from taking up this method and adopting it. They avoid the method completely because of these rumors. Likewise, women already using the device are likely to opt-out due to these same reasons. The accumulation, circulation and spreading of these misconceptions create a contraceptive taboo for the device such that women become stigmatized for choosing the method or the society may frown at these women.

Lastly, these misconceptions are difficult to change; health education aimed at dispelling them only have limited impact and may indirectly stand the risk of strengthening them rather. Therefore, all these conservative beliefs and perceptions about IUDs affect the initial decision of whether or not to choose the device, switch to another method, discontinue or keep using it.

\section{Conclusion}

In line with a study conducted by the International Planned Parenthood Federation, inaccurate information also described as misconceptions are persisting among IUD Users. They have an impact on IUD Usage and can scare women from using the device. They remain barriers to the use of intrauterine devices and are responsible for low patronage in many countries. Inaccurate knowledge on the device might contribute to the reasons why users are still harbouring these conservative beliefs. The dispersion of knowledge and correct information about the device is 
imperative to dispel these misconceptions and increase patronage. Client education must focus on providing accurate information and scientific evidence that will dispel the five influential misconceptions this research has identified to be associated with future usage. Extensive education will help bridge the gap between these beliefs and sexual needs for contraceptives. One of the ways to channel this education is by contraceptive counselling.

Educational intervention should also take a multifaceted approach targeting both the individuals and society. It must be extended to spouses of women with the device to increase social acceptance, ease restrictions and promote supportive partners choice. It should include policymakers, international organizations and civil society leaders who are involved in family planning services. Frequent evaluation and assessment of such educational programs are required to measure how they are desensitizing these misconceptions. Aside from that, healthcare providers have the responsibility to be aware of these misconceptions among users and counter them with accurate knowledge and responses when they arise.

With these interventions in place, women will make choices that are right for them. There will increase social acceptance of Intrauterine Devices irrespective of these myths and hence unintended pregnancies will be reduced. Future areas for research must look at the ideas and emotions that fuel these misconceptions and their sources

\section{Acknowledgements}

We acknowledged the participants voluntarily took part in this study.

\section{Declaration of Competing Interest}

The authors declare that they have no known competing financial interests or personal relationships that could have appeared to influence the work reported in this paper.

\section{Ethical Approval}

Participants gave their written consent to use their anonymous data for statistical purposes. All of them were over 18 years old and voluntarily collaborated without receiving any financial compensation. The procedures were carried out in compliance with the institutional regulations of the National Health Research Ethics Committee, Nigeria (NHREC). Similarly, all procedures adhere to the Helsinki Declaration of 1964, revised in 2013.

\section{References}

[1] Wang, Z., Yuan, W., Tu, X., Liang, H., Miao, M., Cheng, Y., Jin, L., \& Lou, C. (2020). Misconceptions and Beliefs Regarding the Use of Intrauterine Devices for Nulliparous Women Among Chinese Health Care Providers. Journal of Pediatric and Adolescent Gynecology, 33(1), 33-38. doi:10.1016/j.jpag.2019.10.005.

[2] Sedgh, G., Singh, S., \& Hussain, R. (2014). Intended and Unintended Pregnancies Worldwide in 2012 and Recent Trends. Studies in Family Planning, 45(3), 301-314. doi:10.1111/j.1728-4465.2014.00393.x.

[3] Morgan, K. W. (2006). The Intrauterine Device: Rethinking Old Paradigms. Journal of Midwifery \& Women's Health, 51(6), 464-470. doi:10.1016/j.jmwh.2006.06.008.

[4] Chauhan, S. P., Berghella, V., Sanderson, M., Magann, E. F., \& Morrison, J. C. (2006). American College of Obstetricians and Gynecologists practice bulletins: An overview. American Journal of Obstetrics and Gynecology, 194(6), 1564-1572. doi:10.1016/j.ajog.2006.03.001.

[5] Adongo, P. B., Tabong, P. T.-N., Azongo, T. B., Phillips, J. F., Sheff, M. C., Stone, A. E., \& Tapsoba, P. (2014). A Comparative Qualitative Study of Misconceptions Associated with Contraceptive Use in Southern and Northern Ghana. Frontiers in Public Health, 2. doi:10.3389/fpubh.2014.00137.

[6] Thapa, K., Dhital, R., Rajbhandari, S., Acharya, S., Mishra, S., Pokhrel, S. M., Pande, S., Tunnacliffe, E.-A., \& Makins, A. (2019). Factors affecting the behavior outcomes on post-partum intrauterine contraceptive device uptake and continuation in Nepal: A qualitative study. BMC Pregnancy and Childbirth, 19(1), 148. doi:10.1186/s12884-019-2310-y.

[7] Carneiro, M. M., Lira, J., \& Silva-filho, A. L. (2015). Barriers and myths that limit the use of intrauterine contraception in nulliparous women: A survey of Brazilian gynecologists. Fertility and Sterility, 104(3, Supplement), e234. doi:10.1016/j.fertnstert.2015.07.737.

[8] Esposito, C. P., \& LoGiudice, J. (2019). Beliefs and Use of Intrauterine Devices (IUDs) Among Women's Health Care Providers. The Journal for Nurse Practitioners, 15(9), 682-687. doi:10.1016/j.nurpra.2019.05.005.

[9] Payne, J. B., Sundstrom, B., \& DeMaria, A. L. (2016). A Qualitative Study of Young Women's Beliefs about Intrauterine Devices: Fear of Infertility. Journal of Midwifery \& Women's Health, 61(4), 482-488. doi:10.1111/jmwh.12425. 
[10] Elkhateeb, R. R., Kishk, E., Sanad, A., Bahaa, H., Hagazy, A. R., Shaheen, K., Moustafa, E., Fares, H., Gomaa, K., \& Mahran, A. (2020). The acceptability of using IUDs among Egyptian nulliparous women: A cross-sectional study. BMC Women's Health, 20(1), 117. doi:10.1186/s12905-020-00977-9.

[11] Guiahi, M. (2020). Religious refusals to long-acting reversible contraceptives in Catholic settings: A call for evidence. American Journal of Obstetrics and Gynecology, 222(4, Supplement), S869.e1-S869.e5. doi:10.1016/j.ajog.2019.11.1270.

[12] Aniwada, E., James, O., Uchenna, O., Ekuma, O., \& Kelechi, O. (2017). Knowledge, Perception and Misconceptions on Family Planning among Women Living in an Urban Slum in Enugu, Enugu State, Nigeria. Asian Journal of Medicine and Health, 3(2), 1-10. doi:10.9734/AJMAH/2017/32301.

[13] Schwartz, B. I., Alexander, M., \& Breech, L. L. (2020). Levonorgestrel Intrauterine Device Use for Medical Indications in Nulliparous Adolescents and Young Adults. Journal of Adolescent Health. doi:10.1016/j.jadohealth.2020.05.041

[14] Michie, L., Cameron, S. T., Glasier, A., Wellings, K., \& Loudon, J. (2014). Myths and misconceptions about intrauterine contraception among women seeking termination of pregnancy. The Journal of Family Planning and Reproductive Health Care, 40(1), 36-40. doi:10.1136/jfprhc-2012-100497.

[15] Manzouri, L., Aghdak, P., Nematollahi, S., Mansouri, A., Aghababaeian, A., \& Nasiri, S. D. N. D. (2010). Misbelieves about Intra Uterine Device (IUD) in Isfahan, Iran. Journal of Family and Reproductive Health, 169-174.

[16] Hubacher, D. (2014). Intrauterine devices \& infection: review of the literature. The Indian Journal of Medical Research, 140 (Suppl 1), S53.

[17] Lamptey, E., \& Serwaa, D. (2020). The Use of Zipline Drones Technology for COVID-19 Samples Transportation in Ghana.HighTech and Innovation Journal, 1(2), 67-71. doi:10.28991/hij-2020-01-02-03.

[18] J, M., L, G., M, T., M, W., H, S., A, A., C, M., \& P, G. (2020). Exploring contraception myths and misconceptions among young men and women in Kwale County, Kenya. doi:10.21203/rs.2.21058/v2. 